\title{
Epididymitis in Patients with Anorectal Malformations: A Cause for Urologic Concern
}

\author{
Brian A. VanderBrink¹, Bezalel Sivan ${ }^{1}$, Marc A. Levitt ${ }^{1}$, Alberto Peña², Curtis A. Sheldon' ${ }^{1}$ Shumyle Alam¹ \\ ${ }^{1}$ Division of Urology, Division of Pediatric Surgery at Cincinnati Children's Hospital Medical Center, \\ Cincinnati, Ohio USA; ${ }^{2}$ Colorectal Center for Children, Division of Pediatric Surgery at Cincinnati \\ Children's Hospital Medical Center, Cincinnati, Ohio USA
}

\section{ABSTRACT}

Introduction: Epididymitis in patients with anorectal malformation (ARM) represents a unique problem because unlike the general population, an underlying urinary tract problem is frequently identified. We review our experience with epididymitis in ARM population with an emphasis on examining urologic outcomes.

Materials and Methods: We performed a retrospective review of male patients with ARM cared for from 1980 to 2010. Clinical and pathologic variables recorded included age at presentation, recurrence, associated urologic anomalies, incidence of ureteral fusion with mesonephric ductal structures, glomerular filtration rate and urodynamic parameters.

Results: Twenty-six patients were identified with documented episodes of epididymitis. Renal injury was noted in five patients (19\%), all of whom were diagnosed with neurogenic bladder (NGB) several years after anorectoplasty. NGB was found in ten patients (38\%) in our series. Ectopic insertion of ureter into a mesonephric ductal structure was discovered in five patients (19\%). Twelve patients (46\%) had recurrent episodes of epididymitis, with seven of these patients (58\%) being diagnosed with NGB. Two patients in the pubertal group presented with a history of epididymitis and complained of ejaculatory pain.

Conclusion: Epididymitis in a patient with ARM warrants a comprehensive urologic investigation, particularly in recurrent episodes. Attempts at surgical intervention (e.g. vasectomy) should be avoided until functional assessment of the urinary tract has occurred. Failure to recognize this association may lead to potentially avoidable complications and morbidity. Long term urological follow up of these patients is warranted to identify at risk patients and minimize renal deterioration

\section{ARTICLE INFO}

\section{Key words:}

Epididymitis, Anorectal

malformation, Neurogenic

Bladder, Renal Injury

Int Braz J Urol. 2014; 40: 676-82

Submitted for publication:

September 02, 2013

Accepted after revision:

March 14, 2014

\section{INTRODUCTION}

Common causes of acute scrotum in children such as torsion of the spermatic cord or incarcerated hernia require prompt surgery to correct. However other causes such as epididymitis or torsion of the appendix testis typically do not mandate operative intervention. One etiology behind epididymitis in the infant population can be congenital anatomic malformations such as ureteral ectopia into the vas deferens (1). An alternative, and more common cause, is a non-bacterial inflammation secondary to functional disturbance or voiding dysfunction in older children (2). The pathophysiology of voiding dysfunction can lead to external urethral sphincter dyssynergia during 
voiding resulting in elevated urethral pressure and subsequent retrograde urethral-vasal reflux with inflammation and pain $(3,4)$.

Epididymitis in a child with a history of anorectal malformation (ARM) is a previously described phenomenon (5-8). Genitourinary anomalies in the ARM population is also a known entity (9-11) however we have found that the combination of epididymitis and ARM can be an indicator of significant renal and bladder dysfunction. The purpose of this manuscript is to review our institution's experience in the care of children with ARM who have had epididymitis with special emphasis on the need for urologic evaluation to minimize risk of renal injury given this combination of pathology.

\section{MATERIALS AND METHODS}

A retrospective analysis of male patients with ARM treated from 1980 to 2010 was performed with Institutional Review Board approval (IRB\#2008-1317). All cases of epididymitis were identified based upon one or more of the following criteria: history and physical examination suggestive of epididymitis recorded in office/emergency room note, radiographic evidence of epididymitis on ultrasonography or surgical exploration for the acute scrotum with intraoperative findings of epididymitis. No patients were excluded once identified to have an episode of epididymitis based upon the aforementioned clinical criteria.

Clinical and pathologic variables recorded were: age at presentation with epididymitis, laterality of epididymitis, type of anorectal malformation (i.e. level of rectourinary fistula), date of occurrence of epididymitis and its relationship to anorectoplasty (prior to or after surgical repair), presence of tethered spinal cord, associated urinary tract anomalies (i.e. hydronephrosis, vesicoureteral reflux, ureteral ectopia), history of pyelonephritis or urinary tract infection and recurrence of epididymitis. Urologic evaluation included measurement of renal function with determination of glomerular filtration rate and assessment of chronic kidney disease (CKD) stage (12). Glomerular filtration rate (GFR) was measured using DTPA nuclear scan or by measurement of serum Cystatin C (13). Renal ultrasonography and voiding cys- tourethrography were routinely performed in all patients. Nuclear renal scan was utilized to characterize differential renal function or renal scarring in cases of abnormal ultrasonography. Formal urodynamic evaluation of the lower urinary tract was performed in patients with history or radiographic signs of neurogenic bladder. A diagnosis of neurogenic bladder was made based upon urodynamic parameters of uninhibited detrusor contractions, poor compliance of the bladder or incomplete bladder emptying.

All values are reported as median \pm standard deviation. We used a chi-square test to ascertain whether there was a statistical significant difference in frequency of epididymitis between the "high" (recto-bladder neck and recto-prostatic) ARM and "low" (rectobulbar and no fistula) ARM patients. We used a $p$ value of $<0.05$ to be considered statistically significant.

\section{RESULTS}

Twenty six cases of epididymitis were identified during the study period from our ARM patient population (Figure-1). Mean follow up was $15 \pm 11$ years (range $4-25$ years) and three patients were lost to follow-up. Complete radiographic, urodynamic, and follow-up data was available for 10 of the 26 patients. Twelve patients (46\%) had at least one recurrent episode of epididymitis. Mean age at initial episode of epididymitis was $2 \pm 7$ years (range 1 month - 18 years). Epididymitis was encountered during scrotal surgical exploration in ten patients (38\%). Indications of scrotal surgery was concerned over possible testicular torsion $(n=9)$ or drainage of abscess $(n=1)$. A positive urine culture was obtained in five cases treated with antibiotics, however most patients did not have a urine culture collected during the episode.

Ten patients (38\%) suffered from epididymitis prior to their corrective anorectoplasty surgery and after their diverting colostomy. Four of these ten patients had a non-diverting loop colostomy and one of these patients had their stoma converted to a divided end colostomy and mucus fistula after two episodes of epididymitis with no further episodes after that. Eight patients (31\%) presented with epididymitis during or following puberty, several years after their anorectoplasty. Two patients 
Figure 1 - Schematic of the study population. Note the high incidence of neurogenic bladder and renal injury in the recurrent epididymitis patents.

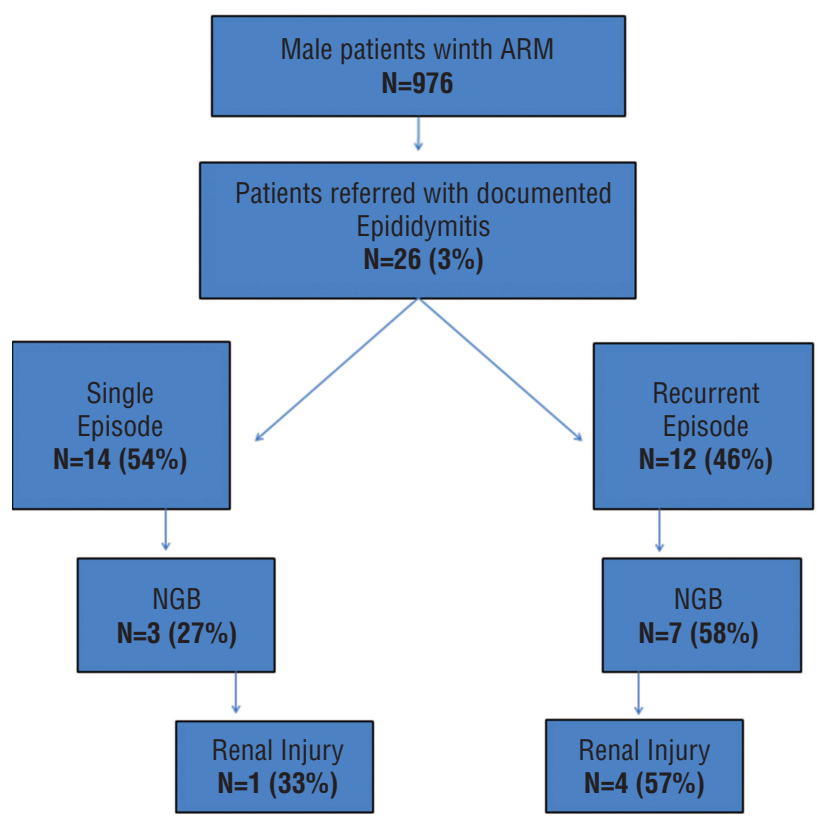

had complaints of ejaculatory pain and erectile dysfunction.

The anatomic level of the ARM fistula was known in 22/26 cases by review of the operative note from anorectoplasty or distal colostogram. When known, the level of fistula between the rectum and urinary tract was subjectively classified as "high" (recto-bladder neck n=9; and recto-prostatic; $n=8)$ in seventeen (77\%) patients whereas five (23\%) were classified as "low" lesions (recto-bulbar; $\mathrm{n}=4$ and no fistula; $\mathrm{n}=1$ ). Ninety percent of patients who suffered recurrent epididymitis had a "high" ARM. A correlation was found between a "high" ARM and the frequency of epididymitis compared to "low" ARM patients ( $\mathrm{p}=0.02)$.

Associated urological abnormalities were presented in 22 patients (85\%) (Table-1). The most common urologic associated anomaly was vesicoureteral reflux in thirteen patients (50\%). Seventeen ureters refluxed in these thirteen patients with grade of reflux known in seven patients with twelve refluxing ureters. The known grades of reflux in these seventeen refluxing ureters were: Grade 1 $(n=1)$, Grade $2(n=8)$, Grade $3(n=3)$, Grade $4(n=2)$ and Grade $5(n=3)$. An ectopic ureter inserting into vas deferens was identified in five patients (19\%). Six patients underwent surgery for either an ectopic location $(n=5)$ or persistent reflux despite maximal medical therapy $(n=1)$. Two of the five patients with the ectopic ureteral location to the vas deferens underwent nephroureterectomy due to poor function of the renal moiety while the remaining three patients underwent reimplantation. Cystoscopy was performed in 18 patients, however most patients had their cystoscopy performed in conjunction with their original anorectoplasty rather than following the episode of epididymitis. Cystoscopy revealed the verumontanum located in bladder neck in one patient who presented with ejaculatory dysfunction (Figure-2).

A spinal MRI was performed in 20 patients within the epididymitis cohort. A tethered spinal cord was identified in five patients and all were submitted to spinal surgery to correct the tethering. Formal urodynamics was performed in eleven patients (42\%) with evidence of neurogenic bladder seen on the urodynamic study in ten of these patients (91\%). Urodynamic findings included detrusor instability, impaired compliance or diminished expected bladder capacity. Nuclear renal scan was performed in 10 patients. Five of the ten (50\%) patients with diagnosis of neurogenic bladder based upon urodynamics had evidence of renal injury upon measurement of GFR (Table-2). Four of the five (80\%) patients had CKD stage 2 or higher with three of these having CKD Stage 3 (Table 3). None of the patients with CKD Stage 3 had evidence of renal dysplasia on imaging with only one of them having evidence of ureter inserting into vas deferens.

\section{DISCUSSION}

Historically epididymitis in the general pediatric population has led to the recommendation to investigate for an anomalous connection between the urinary tract and Wolffian structures (14). This is a rare finding given the relative frequency of acute scrotum due to epididymitis (15). In contrast, we present our series of males with a history of ARM presenting with clinical signs 
Table 1 - Incidence of Urinary Tract Abnormalities in Patients with ARM and Epididymitis.

\begin{tabular}{lc}
\hline Urinary Tract Anomaly & Number (Percentage) \\
\hline Hydronephrosis due Obstruction & $13(50 \%)$ \\
Reflux & $13(50 \%)$ \\
Renal Dysplasia & $8(31 \%)$ \\
Ectopic ureter into Vas & $5(19 \%)$ \\
Renal Agenesis & $4(15 \%)$ \\
Urethral Anomalies & $6(23 \%)$ \\
\hline
\end{tabular}

of epididymitis that had high degree of urologic dysfunction and nearly 20\% incidence of ectopic ureter. A significant proportion of patients in our series also had evidence of renal injury or neurogenic bladder. Therefore we strongly advocate for a comprehensive urologic evaluation to elucidate the etiology behind the epididymitis in the ARM patient population.

Similar to other published series, we found a higher incidence of recto-bladder neck or recto-prostatic fistulas relative to other types of recto-urinary fistulas in our series of males with ARM and epididymitis (5-8). One explanation of this

Figure 2 - Cystoscopic view of verumontanum located in bladder neck from an adult ARM patient that had suffered from recurrent epididymitis and painful ejaculation.

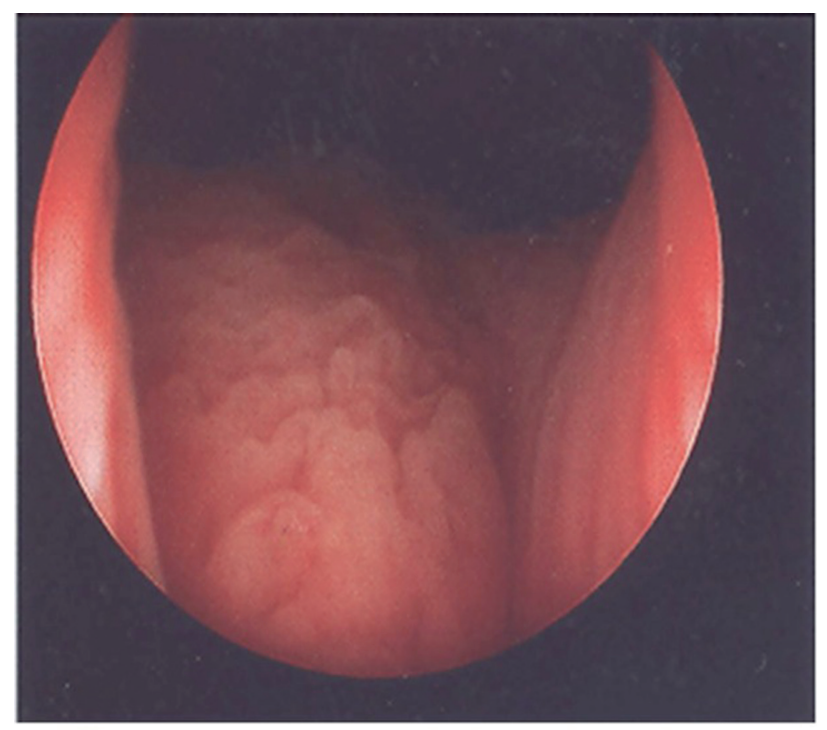

observation is the unavoidable surgical dissection around the bladder neck/seminal vesicles in the case of rectobladder neck or proximal recto-prostatic fistula may have contributed to the bladder dysfunction leading to epididymitis. However in our series we did not have preoperative urodynamics to make the claim that any degree of observed bladder dysfunction was acquired rather than preexistent. However, abnormal urodynamics can be seen following posterior sagittal anorectoplasty (PSARP) when they were absent preoperatively. Boemer et al. in their series of 32 children with ARM, three children (9\%), had evidence of abnormal urodynamics following PSARP with two of these being de novo (16). The abnormalities observed were either parasympathetic innervation with resultant loss of detrusor contractility in two patients or the remaining patient had mixed parasympathetic and sympathetic denervation of the bladder with associated bladder neck incompetence. Upon follow-up, the authors reported this last patient demonstrated evidence of bladder deterioration in the form of detrusor hyperreflexia and dyssynergia with functional infravesical obstruction. The authors attributed the abnormal urodynamics to a subset of ARM patients where a combined posterior sagittal and trans-abdominal dissection is needed or the presence of rectourethral fistula (16). The neuroanatomy of males with rectourethral fistula due to ARM has demonstrated that both pelvic splanchnic nerves come into close proximity at the level of the distal rectum and are only separated by the thin fistula itself (17). Other authors have also observed a change in urodynamics after extensive dissection needed for correction of ARM $(18,19)$. Warne et al. demonstrated 60\% incidence of bladder dysfunction at presentation in 20 patients with ARM, however a change in bladder function following reconstructive surgery was noted in only one of the patients who had a recto-bladder neck fistula repaired with a combined posterior sagittal and abdominal approach (18).

An abnormal sacrum with abnormal spinal cord can have prognostic information with respect to achieving fecal continence following PSARP (20). Conflicting reports exist when using these radiographic characteristics to predict lower 
Table 2 - Renal Outcomes in patients who had Epididymitis and Anorectal Malformation with Neurogenic Bladder.

\begin{tabular}{lccc}
\hline Fistula Location & $\begin{array}{c}\text { Ectopic Ureter inserting into Vas } \\
\text { Deferens }\end{array}$ & $\begin{array}{c}\text { Recurrent Epididymitis } \\
\text { R-BN }\end{array}$ & $\begin{array}{c}\text { Glomerular Filtration Rate (mL/ } \\
\left.\mathrm{min} / 1.73 \mathrm{~m}^{2}\right)\end{array}$ \\
R-BN & Yes & Yes & 124 \\
R-BN & No & Yes & 38 \\
R-P & Yes & Yes & 48 \\
No Fistula & No & No & 108 \\
R-P & Yes & Yes & 129 \\
R-BN & No & Yes & 162 \\
R-BN & Yes & No & 35 \\
R-B & No & Yes & 97 \\
R-BN & No & No & 82 \\
\hline
\end{tabular}

Abbreviations: R-BN = Recto bladder neck fistula; $\mathbf{R}-\mathbf{P}=$ Rectoprostatic fistula; $\mathbf{R}-\mathbf{B}=$ Rectobulbar

urinary tract dysfunction in ARM (21-24). In our select series of males with epididymitis we did not see an association between the existence of neurogenic bladder and either sacral or spinal cord radiographic abnormality. Half of our patients with neurogenic bladder did not have evidence of spinal cord tethering. A similar lack of association for sacral abnormalities existed. These are very similar results to those recently reported by Stathopoulos et al. (21). They evaluated 80 children with MRI imaging of the spine and preoperative urodynamics to assess for presence of neuropathic bladder and, if identified, whether any association between vertebral or spinal cord anomalies could be discovered. The authors found abnormal urodynamics suggestive of neurogenic bladder in 14 children (18\%). However no association could be made as seven children (50\%) had no evidence of vertebral or spinal cord anomalies while seven (50\%) did. Taskinen et al. utilized similar screening testing in thirty children with ARM following their reconstructive surgery because of either fecal or urinary incontinence (22). When the spinal cord was normal based on MRI, 54\% of the patients had abnormal urodynamic findings but when the spinal cord was abnormal, 59\% had abnormal urodynamics. When the bony spine was normal, $33 \%$ of the patients had an abnormal spinal cord but when the bony spine was abnormal, 69\% had an abnormal spinal cord. Therefore our results add to the literature in highlighting the need for formal urodynamic evaluation regardless of spinal cord or sacral imaging, especially in cases of epididymitis.

The presenting symptoms of epididymitis varied in our series. Fever and scrotal swelling were the symptoms most frequently observed, however ejaculatory dysfunction was only seen in the adolescent and adult patients. This occurred in two (8\%) of the 26 patients in our cohort. This symptom was reported by Konuma et al. in males with ARM over the age of twenty. In their series of seventeen men over the age of 20 years old ejaculatory incompetence, defined as patients who had no sperm in their urine or the patients who had no chance to assess their urine, was seen in 5 patients (29\%) and retrograde ejaculation in 2 patients (12\%). Spinal cord anomalies varied from myelodysplastic features in some to normal radiographic findings in others, thus the relationship between sacral anomaly and ejaculatory function remained unclear. 
Table 3 - Urodynamic Findings in patients who had Epididymitis and Anorectal Malformation.

\begin{tabular}{lcccccccc}
\hline $\begin{array}{l}\text { Level } \\
\text { of } \\
\text { Fistula }\end{array}$ & $\begin{array}{c}\text { Sacral } \\
\text { Abnormality }\end{array}$ & $\begin{array}{c}\text { Recurrent } \\
\text { Epididymitis }\end{array}$ & $\begin{array}{c}\text { Tethered } \\
\text { Cord }\end{array}$ & $\begin{array}{c}\text { Cystometric } \\
\text { Capacity }(\mathrm{mL})\end{array}$ & $\begin{array}{c}\text { Uninhibited } \\
\text { Detrusor } \\
\text { Contractions }\end{array}$ & $\begin{array}{c}\text { Maximum } \\
\text { Detrusor Pressure } \\
\left(\mathrm{cm} \mathrm{H}_{2} \mathrm{O}\right)\end{array}$ & $\begin{array}{c}\text { Post Void } \\
\text { residual } \\
(\mathrm{mL})\end{array}$ & $\begin{array}{c}\text { GFR }(\mathrm{mL} / \\
\left.\mathrm{min} / 1.73 \mathrm{~m}^{2}\right)\end{array}$ \\
\hline R-BN & - & + & - & 640 & & 40 & 640 & 124 \\
R-BN & - & + & - & 327 & - & 10 & 150 & 38 \\
R-BN & + & + & + & 360 & + & 133 & 291 & 48 \\
R-P & - & - & - & 90 & + & 43 & 90 & 108 \\
Unk & - & + & + & 580 & + & 15 & 95 & 129 \\
R-P & - & + & - & 516 & + & 38 & 78 & 162 \\
Unk & + & - & + & 360 & - & 69 & 360 & 35 \\
R-BN & + & + & - & 200 & + & 75 & 60 & 97 \\
Unk & - & - & - & 627 & - & 12 & 150 & 121 \\
R-B & + & + & - & 250 & + & 55 & 59 & 82 \\
R-BN & - & + & + & 350 & + & 72 & 98 & 49 \\
\hline
\end{tabular}

Abbreviations: GFR = glomerular filtration rate; $\mathbf{R}-\mathbf{B N}=$ Recto bladder neck fistula; $\mathbf{R}-\mathbf{P}=$ Rectoprostatic fistula; $\mathbf{R}-\mathbf{B}=$ Rectobulbar $;$ Unk = Unknown

Another important cause of epididymitis may be the ectopic location of the verumontanum near the bladder neck. We speculate that this congenital ectopic location may lead to voiding into the vas deferens and resultant epididymal irritation/inflammation. Finally adding to the difficulty in reaching any definitive conclusions is that surgical techniques in these patients operated on years ago varied as refinements in technique inevitably occur over a three decade span. Therefore we can only speculate that the observed ejaculatory dysfunction is multifactorial and due to the same mechanism contributing to neurogenic bladder.

There are certain limitations to our study and we acknowledge the impact they may have on our results and conclusions. First and foremost is the retrospective nature of this study with its inherent limitations and biases. We also did not have complete urodynamic and GFR data on every patient yet did find a significant prevalence of chronic kidney disease in the subset where this information was available. Despite these limitations, we felt compelled to draw attention to physicians that the diagnosis of epididymitis in a child with ARM should not be trivialized. On the contrary this may be a harbinger of significant urologic dysfunction and a reminder that ongoing urologic surveillance is needed in all patients with ARM because of the risk of delayed development renal and bladder dysfunction.

\section{CONCLUSIONS}

ARM patients diagnosed with epididymitis in our series had a high incidence of neurogenic bladder and renal injury. A significant minority also had evidence of pathologic connection between the ectopic ureter and Wolffian structure. Patients that presented in adolescence or as adult patients that suffer from recurrent epididymitis may develop dysfunctional or painful ejaculation.

We advocate for performing a full urological work-up to evaluate for underlying cause of the epididymitis, especially in recurrent cases. Long term follow-up should be performed due to the possibility of NGB and the risk of developing 
renal injury. Definitive surgical correction (e.g. vasectomy) should be avoided until functional aspects have been addressed.

\section{REFERENCES}

1. Merlini E, Rotundi F, Seymandi PL, Canning DA. Acute epididymitis and urinary tract anomalies in children. Scand $\mathrm{J}$ Urol Nephrol. 1998;32:273-5.

2. Bukowski TP, Lewis AG, Reeves D, Wacksman J, Sheldon CA. Epididymitis in older boys: dysfunctional voiding as an etiology. J Urol. 1995;154(2 Pt 2):762-5.

3. Franco I. Functional bladder problems in children: pathophysiology, diagnosis, and treatment. Pediatr Clin North Am. 2012;59:783-817.

4. Thind P, Gerstenberg TC, Bilde T. Is micturition disorder a pathogenic factor in acute epididymitis? An evaluation of simultaneous bladder pressure and urine flow in men with previous acute epididymitis. J Urol. 1990;143:323-5.

5. Raveenthiran V, Sam CJ. Epididymo-orchitis complicating anorectal malformations: collective review of 41 cases. J Urol. 2011;186:1467-72.

6. Kiyan G, Dagli TE, Iskit SH, Tugtepe H. Epididymitis in infants with anorectal malformation. Eur Urol. 2003;43:576-9.

7. Oğuzkurt $P$, Tanyel FC, Büyükpamukçu N. Acute scrotum due to edidymo-orchitis associated with vasal anomalies in children with anorectal malformations. J Pediatr Surg. 1998;33:1834-6.

8. Rubio Cordero JL, Nuñez Nuñez R, Blesa Sanchez E. Ano-rectal malformation and recurring orchioepididymitis in infants. Eur $\mathrm{J}$ Pediatr Surg. 1994;4:46-8.

9. Goossens WJ, de Blaauw I, Wijnen MH, de Gier RP, Kortmann $B$, Feitz WF. Urological anomalies in anorectal malformations in The Netherlands: effects of screening all patients on long-term outcome. Pediatr Surg Int. 2011;27:1091-7.

10. McLorie GA, Sheldon CA, Fleisher M, Churchill BM. The genitourinary system in patients with imperforate anus. J Pediatr Surg. 1987;22:1100-4.

11. Misra D, Mushtaq I, Drake DP, Kiely EM, Spitz L. Associated urologic anomalies in low imperforate anus are capable of causing significant morbidity: a 15-year experience. Urology. 1996;48:281-3.

12. Hogg RJ, Furth S, Lemley KV, Portman R, Schwartz GJ, Coresh $J$, et al. National Kidney Foundation's Kidney Disease Outcomes Quality Initiative clinical practice guidelines for chronic kidney disease in children and adolescents: evaluation, classification, and stratification. Pediatrics. 2003;111(6 Pt 1):1416-21.

13. Bökenkamp A, Domanetzki M, Zinck R, Schumann G, Byrd D, Brodehl J. Cystatin C--a new marker of glomerular filtration rate in children independent of age and height. Pediatrics. 1998;101:875-81.
14. Al-Taheini KM, Pike J, Leonard M. Acute epididymitis in children: the role of radiologic studies. Urology. 2008;71:826-9; discussion 829.

15. McAndrew HF, Pemberton R, Kikiros CS, Gollow I. The incidence and

16. investigation of acute scrotal problems in children. Pediatr Surg Int. 2002;18:435-7.

17. Boemers TM, Bax KM, Rövekamp MH, van Gool JD. The effect of posterior sagittal anorectoplasty and its variants on lower urinary tract function in children with anorectal malformations. J Urol. 1995;153:191-3.

18. Scott JE. The anatomy of the pelvic autonomic nervous system in cases of high imperforate anus. Surgery. 1959;45:1013-28.

19. Warne SA, Godley ML, Wilcox DT. Surgical reconstruction of cloacal malformation can alter bladder function: a comparative study with anorectal anomalies. J Urol. 2004;172(6 Pt 1):237781; discussion 2381.

20. Jindal B, Grover VP, Bhatnagar V. The assessment of lower urinary tract function in children with anorectal malformations before and after PSARP. Eur J Pediatr Surg. 2009;19:34-7.

21. Peña A. Anorectal malformations. Semin Pediatr Surg. 1995:4:35-47.

22. Stathopoulos E, Muehlethaler V, Rais M, Alamo L, Dushi G, Frey $P$, et al. Preoperative assessment of neurovesical function in children with anorectal malformation: association with vertebral and spinal malformations. J Urol. 2012;188:943-7.

23. Taskinen S, Valanne L, Rintala R. Effect of spinal cord abnormalities on the function of the lower urinary tract in patients with anorectal abnormalities. J Urol. 2002;168:1147-9.

24. Mosiello G, Capitanucci ML, Gatti C, Adorisio 0, Lucchetti MC Silveri $\mathrm{M}$, et al. How to investigate neurovesical dysfunction in children with anorectal malformations. J Urol. 2003;170(4 Pt 2):1610-3.

25. Kiliç N, Emir H, Sander S, Eliçevik M, Celayir S, Söylet Y. Comparison of urodynamic investigations before and after posterior sagittal anorectoplasty for anorectal malformations. J Pediatr Surg. 1997;32:1724-7.

26. Konuma K, Ikawa H, Kohno M, Okamoto S, Masuyama H, Fukumoto $\mathrm{H}$. Sexual problems in male patients older than 20 years with anorectal malformations. J Pediatr Surg. 2006;41:306-9.

Correspondence address: Brian A. VanderBrink, MD Cincinnati Children's Hospital Medical Center Division of Urology MLC 5037 3333 Burnet Avenue Cincinnati, OH 45229 Phone: 513-636-4975 E-mail: brian.vanderbrink@cchmc.org 